\title{
Wichtiger Hinweis
}

Dieses Buch besteht aus drei Bänden mit insgesamt 21 Kapiteln. Zu jedem Kapitel gehören Ergänzungen.

Die Kapitel bilden für sich eine Einheit und können unabhängig von den Ergänzungen gelesen werden.

Die Ergänzungen schließen jeweils an das entsprechende Kapitel an und sind in der Kopfzeile durch das Zeichen • gesondert gekennzeichnet. Sie beginnen mit einer kurzen Inhaltsübersicht, die als Leseanleitung verstanden werden kann.

Die Abschnitte in den Ergänzungen sind von verschiedener Art: Einige erleichtern das Verständnis des zugehörigen Kapitels oder dienen der weiteren Präzisierung; andere befassen sich mit konkreten physikalischen Anwendungen oder verweisen auf bestimmte Teilgebiete der Physik. Ein Abschnitt enthält schließlich die Aufgaben zum betreffenden Kapitel. Es wird nicht erwartet und ist auch nicht immer zweckmäßig, die Ergänzungen in der angegebenen Reihenfolge zu erarbeiten.

In den beiden ersten Bänden wird gelegentlich auf die Anhänge I bis III verwiesen. Diese befinden sich am Ende des zweiten Bands. 
